\title{
Flow rate-pressure drop relation for deformable shallow microfluidic channels
}

\author{
Ivan C. Christov ${ }^{1,3} \dagger$, Vincent Cognet ${ }^{1,2}$, Tanmay C. Shidhore ${ }^{3}$ and \\ Howard A. Stone ${ }^{1} \dagger$ \\ ${ }^{1}$ Department of Mechanical and Aerospace Engineering, Princeton University, \\ Princeton, New Jersey 08544, USA \\ ${ }^{2}$ École Normale Supérieure de Cachan, Cachan Cedex, France \\ ${ }^{3}$ School of Mechanical Engineering, Purdue University, West Lafayette, Indiana 47907, USA
}

(Received ?; revised ?; accepted ?. Today is 4 January 2018)

Laminar flow in devices fabricated from soft materials causes deformation of the passage geometry, which affects the flow rate-pressure drop relation. For a given pressure drop, in channels with narrow rectangular cross-section, the flow rate varies as the cube of the channel height, so deformation can produce significant quantitative effects, including nonlinear dependence on the pressure drop [Gervais, T., El-Ali, J., Günther, A. \& Jensen, K. F. 2006 Flow-induced deformation of shallow microfluidic channels. Lab Chip 6, 500507]. Gervais et al. proposed a successful model of the deformation-induced change in the flow rate by heuristically coupling a Hookean elastic response with the lubrication approximation for Stokes flow. However, their model contains a fitting parameter that must be found for each channel shape by performing an experiment. We present a perturbation approach for the flow rate-pressure drop relation in a shallow deformable microchannel using the theory of isotropic quasi-static plate bending and the Stokes equations under a lubrication approximation (specifically, the ratio of the channel's height to its width and of the channel's height to its length are both assumed small). Our result contains no free parameters and confirms Gervais et al.'s observation that the flow rate is a quartic polynomial of the pressure drop. The derived flow rate-pressure drop relation compares favorably with experimental measurements.

\section{Introduction}

Fluid-structure interactions in low-Reynolds-number flows occur at many scales and across various physical contexts (Bodnár et al. 2014; Duprat \& Stone 2016). For example, microfluidic channels created using polymers such as poly(dimethylsiloxane) (PDMS) are soft (Lötters et al. 1997; Xia \& Whitesides 1998). As a result, deformation away from their rectangular cross-sectional molding should be expected, and indeed has been observed under typical flow conditions (Holden et al. 2003; Gervais et al. 2006; Seker et al. 2009; Hardv et al. 2009; Cheung et al. 2012; Raj \& Sen 2016; Raj et al. 2017). It is well known that, in a laminar viscous flow through a channel of arbitrary but fixed crosssection specified a priori, the pressure drop $\Delta p$ across the channel is proportional to the volumetric flow rate $q$ through it (Happel \& Brenner 1983; Sutera \& Skalak 1993; Bruus 2008). Moreover, the proportionality constant, which is termed the hydrodynamic resistance, is only a function of the fluid's viscosity and the geometry of the channel (Happel \& Brenner 1983; Bruus 2008). While such a $q-\Delta p$ relation is valid for sufficiently low flow rates and low pressure drops, a markedly nonlinear regime due to fluid-structure

$†$ Email addresses for correspondence: christov@purdue.edu, hastone@princeton.edu 
interactions has been observed experimentally at higher values of $q$ or $\Delta p$ (Gervais et al. 2006; Seker et al. 2009; Hardy et al. 2009; Cheung et al. 2012). Gervais et al. (2006) proposed a simple model (with one fitting parameter) of the flow rate-pressure drop relationship in a soft shallow microchannel. Based on the assumption of a Hookean response of the elastic channel walls to the fluid pressure, Gervais et al. (2006) related the channel shape to the hydrodynamic pressure, and inserted this relation into the standard relationship from lubrication theory between the pressure gradient and the flow rate. While this approach was successful in describing experimental measurements, it is not a complete theory. In the present work, we use asymptotic analysis to find the flow ratepressure drop relation in the bending-dominated regime of channel deformation, without any fitting parameters.

Obtaining analytical expressions, even approximate ones, for such a "generalized Poiseuille's law" is of importance for the design and construction of microfluidic devices (Schomburg 2011; Sollier et al. 2011; Ozsun et al. 2013; Anoop \& Sen 2015; Raj \& Sen 2016; Raj et al. 2017; Gomez et al. 2017), where the compliance of soft polymeric materials allows higher throughput than their rigid counterparts. In turn, this degree of freedom makes it possible to manufacture various valves, pumps and self-regulating fluid control elements for lab-on-a-chip technologies (see, e.g., Squires \& Quake 2005, Section III.C). Similarly, on the basis of the flow-deformation coupling, Holden et al. (2003) proposed using deformable microchannels as microfluidic diffusion diluters. Beyond microfluidics, a flow rate-pressure drop relation is also necessary for, e.g., upscaling deformable porous media (Iliev et al. 2008), modeling multiphase fluid-structure interactions in industrial piping and turbomachinery (Ando et al. 2011), and understanding biofluid mechanics of blood vessels (Pedley 1980; Grotberg \& Jensen 2004).

In fact, similar types of fluid-structure interaction problems have a history in biofluid mechanics, before the modern advent of microfluidics. For example, Fung, in his Biomechanics textbook (Fung 1997, §3.4), derived a flow rate-pressure drop relation for steady, laminar flow in an elastic tube starting from Poiseuille's relation for a pipe of uniform radius and substituting an axially-varying radius, which is found as a solution to Hooke's law accounting for only circumferential strains. An important consequence of this result is that $q$ is a nonlinear function of $\Delta p$, which has significant implications for biofluid mechanics of flow through soft tubes (Rubinow \& Keller 1972; Grotberg \& Jensen 2004), specifically in the study of collapsible blood vessels and constrictions (Conrad 1969; Katz et al. 1969; Pedlev 1980; Kizilova et al. 2012). In this context, a perturbative approach to the derivation of the related "tube laws" (relationships between pressure drop and cross-sectional area of a tube) from shell theory has proven fruitful (Whittaker et al. 2010).

Here, we are interested in the steady-state response of a a microchannel due to flow through it. Nevertheless, the transient deformation problem for a microchannel has been studied experimentally (Dendukuri et al. 2007; Panda et al. 2009), showing that the characteristic response time can be found in terms of the system's geometric parameters, the fluid's viscosity and the elastic solid's Young's modulus. A lubrication model captures the bulk of the transient response observed in experiments (Panda et al. 2009), thereby verifying the scaling analysis of Dendukuri et al. (2007); Mukherjee et al. (2013) extended the one-dimensional lubrication model of Panda et al. (2009) to account for electroosmotic flows. It is of interest to account for the effect of fluid-structure interactions in such flows because, for example, flow-wise cross-sectional variations affect electrokinetics and increase electrolyte dispersion (Ghosal 2002; Bahga et al. 2012) and can be used to improve the sensitivity of impedance-based flow rate measurements (Niu et al. 2017). More recently, Elbaz \& Gat (2014, 2016) analyzed, using perturbation methods, sev- 
eral problems of axisymmetric axial viscous flows in soft cylinders, obtaining closed-form leading-order solutions of the transient response of the elastic shell and the corresponding time evolution of the fluid pressure.

Beyond one-dimensional models, fewer works have considered the full three-dimensional response of the microchannel. Gervais et al. (2006) performed steady-state fluid-structure interaction simulations, while Ozsun et al. (2013) simulated only the fluid flow in the deformed channel by using their experimentally measured wall deformation to set the geometry. Meanwhile, Chakraborty et al. (2012) employed a three-dimensional computational model, solving numerically the coupled equations of fluid mechanics and elasticity. However, Chakraborty et al. (2012) showed that a two-dimensional model based on assuming the elastic wall is an infinitely wide elastic beam of finite thickness is a reasonable approximation to the three-dimensional deformations observed in experiments. Thus, it has been established that there is value in lower-dimensional models, especially if the models can be analyzed completely.

To this end, in this paper, we begin by deriving the governing equations of lubrication theory for long, shallow microchannels in 2, via a leading-order asymptotic solution of the Stokes equations. Then, unlike the textbook problem of a conduit of fixed shape, in $\$ 3$ we couple the equations from 92 to the governing equations of Kirchhoff-Love plate theory. On the basis of the leading-order solutions for the streamwise velocity component and for the plate deformation, in 84 a fitting-parameter-free expression for the flow rate-pressure drop relation for a long, shallow microchannel is derived. In \$5 the analytical expressions derived are illustrated for a range of values of the dimensionless parameters, and shown to compare favorably with experimental measurements. Finally, conclusions are stated in 6 . In Appendix A some explicit formulæ for the velocity profile and the pressure as functions of the axial coordinate are presented for the special case of a microchannel with a "stiff" top wall. Meanwhile, Appendix B includes further mathematical details regarding the effect of the lateral sidewalls on our analysis.

\section{Lubrication approximation for shallow deformable channels}

We consider a channel of length $\ell$, width $w$ and height $h$, where $h \ll w \ll \ell$. The upper wall of the channel is soft and deformable, as is the case when a rigid channel is sealed by a thin elastic film. The flow is in the positive $z$-direction. Due to the normal stresses from the flow on the walls, the (soft) top wall of the channel deforms out of the $(x, z)$-plane in the positive $y$-direction, so that the steady shape of the channel's top wall is given by $y=h(x, z)=h_{0}+u(x, z)$, as shown in figure1 $(a)$. For the moment, we make no assumption on the magnitude of the displacement, however, we expect that $u>0$ and $u \ll w$ for the types of problems of interest herein.

Now, consider the incompressible flow of a Newtonian fluid of density $\varrho$ and viscosity $\mu$. As is typical for microfluidic devices (Stone et al. 2004; Squires \& Quake 2005; Bruus 2008), we are interested in the limit in which the effective Reynolds number is small, i.e., $\epsilon R e=\left(h_{0} / \ell\right) \varrho \mathcal{V}_{c} h_{0} / \mu \ll 1$, where $\mathcal{V}_{c}$ is a characteristic axial velocity and $\epsilon=h_{0} / \ell \ll 1$. In this limit, fluid inertia is negligible compared to viscous stresses, and the flow is governed by the Stokes equations (Happel \& Brenner 1983; Bruus 2008):

$$
\mathbf{0}=-\nabla p+\mu \nabla^{2} \boldsymbol{v}, \quad \boldsymbol{\nabla} \cdot \boldsymbol{v}=0,
$$

where $\boldsymbol{v}=\left(v_{x}, v_{y}, v_{z}\right)$ and body forces have been neglected. The flow is also subject to 


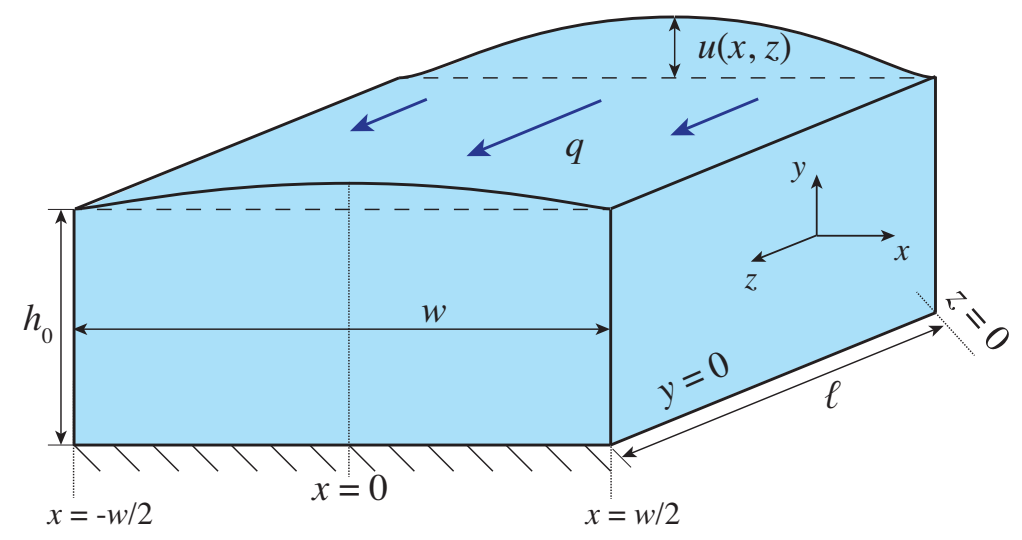

Figure 1: Three-dimensional schematic of the deformed geometry of a channel of length $\ell$ with an initially rectangular cross-section $\left(w \times h_{0}\right)$. The volumetric flow rate is denoted by $q$, and $u(x, z)$ is the top wall's deformation (the remaining walls are assumed rigid). Although not shown in this schematic, the top wall has a thickness $t$.

the no-slip and no-penetration boundary conditions along the walls of the channel:

$$
\boldsymbol{v}=\mathbf{0} \quad \text { at } \quad\left\{\begin{array}{l}
y=0, h(x, z), \\
x= \pm w / 2
\end{array}\right.
$$

Note that the velocity vanishes along $y=h$, rather than being $v_{x}=d h / d t$, etc., because we have already assumed the fluid flow and the structural deformation to be steady (i.e., independent of time).

Let us introduce the dimensionless variables

$$
\begin{array}{r}
x=w X, \quad y=h_{0} Y, \quad z=\ell Z, \quad h(x, z)=h_{0} H(X, Z), \quad u(x, z)=u_{\mathrm{c}} U(X, Z), \\
v_{x}(x, y, z)=\frac{\epsilon}{\delta} \mathcal{V}_{\mathrm{c}} V_{X}(X, Y, Z), \quad v_{y}(x, y, z)=\epsilon \mathcal{V}_{\mathrm{c}} V_{Y}(X, Y, Z), \\
v_{z}(x, y, z)=\mathcal{V}_{\mathrm{c}} V_{Z}(X, Y, Z), \quad p(x, y, z)=\Delta p P(X, Y, Z),
\end{array}
$$

where $\Delta p, u_{\mathrm{c}}$ and $\mathcal{V}_{\mathrm{c}}$ are a characteristic pressure scale, a characteristic top wall deformation scale, and a characteristic axial velocity scale, respectively; we have also defined $\delta:=h_{0} / w$ and $\epsilon:=h_{0} / \ell$. The characteristic top wall deformation $u_{\mathrm{c}}$ is not an independent parameter and will be determined below, upon scaling the governing equations. First, consider the continuity equation $(2.1 \mathrm{~b})$, which becomes

$$
\frac{\partial V_{X}}{\partial X}+\frac{\partial V_{Y}}{\partial Y}+\frac{\partial V_{Z}}{\partial Z}=0
$$

By convention, we sought a balance in the continuity equation, which is how the velocity scales in (2.3) were set.

A microchannel can be operated in one of two regimes: either the pressure drop $\Delta p=$ $p(0)-p(\ell)$ is imposed, where we assume that the outlet is always open to the atmosphere so we set $p(\ell)=0$ (then, the inlet pressure is $p(0)=\Delta p$ ), or the flow rate $q$ is imposed, which is equivalent to imposing the mean flow velocity $\left\langle v_{z}\right\rangle \propto q /\left(h_{0} w\right)$. In both cases, $\Delta p$ and $\mathcal{V}_{\mathrm{c}}$ are not independent scales but coupled by the flow physics. In the first case, the characteristic velocity scale is set by pressure drop $\Delta p: \mathcal{V}_{\mathrm{c}}=h_{0}^{2} \Delta p /(\mu \ell)$; in the second case, the pressure drop is set by the characteristic axial velocity scale $\mathcal{V}_{\mathrm{c}}=\left\langle v_{z}\right\rangle$ : 
$\Delta p=\mu\left\langle v_{z}\right\rangle \ell / h_{0}^{2}$. In either case, (2.1 a) adopts the same form:

$$
\begin{aligned}
\epsilon^{2} \delta^{2} \frac{\partial^{2} V_{X}}{\partial X^{2}}+\epsilon^{2} \frac{\partial^{2} V_{X}}{\partial Y^{2}}+\epsilon^{4} \frac{\partial^{2} V_{X}}{\partial Z^{2}} & =\delta^{2} \frac{\partial P}{\partial X} \\
\epsilon^{2} \delta^{2} \frac{\partial^{2} V_{Y}}{\partial X^{2}}+\epsilon^{2} \frac{\partial^{2} V_{Y}}{\partial Y^{2}}+\epsilon^{4} \frac{\partial^{2} V_{Y}}{\partial Z^{2}} & =\frac{\partial P}{\partial Y} \\
\delta^{2} \frac{\partial^{2} V_{Z}}{\partial X^{2}}+\frac{\partial^{2} V_{Z}}{\partial Y^{2}}+\epsilon^{2} \frac{\partial^{2} V_{Z}}{\partial Z^{2}} & =\frac{\partial P}{\partial Z} .
\end{aligned}
$$

Above, we assumed that the channel is long and thin, which leads to a natural ordering of the small (dimensionless) parameters:

$$
0<\epsilon \ll \delta \ll 1 \text {. }
$$

We are interested in the leading-order asymptotic behavior under the ordering (2.6). From (2.5), we have

$$
\frac{\partial^{2} V_{Z}}{\partial Y^{2}}=\frac{\partial P}{\partial Z}, \quad 0=\frac{\partial P}{\partial Y}, \quad 0=\frac{\partial P}{\partial X},
$$

which is the familiar lubrication approximation. From $(2.7 b, c)$, we first infer that the leading-order pressure does not depend on the cross-sectional coordinates $(X, Y)$. Then, after integrating (2.7 a) and enforcing the no-slip boundary condition from (2.2), i.e., $V_{Z}(X, 0, Z)=V_{Z}(X, H(X, Z), Z)=0 \forall X, Z$, the "standard" lubrication theory result for the axial velocity follows:

$$
V_{Z}(X, Y, Z)=-\frac{1}{2} \frac{\mathrm{d} P}{\mathrm{~d} Z}(H(X, Z)-Y) Y .
$$

We remind the reader that $\mathrm{d} P / \mathrm{d} Z<0$ in our convention. This leading-order axial velocity does not satisfy the no-slip boundary condition at the side walls $(X= \pm 1 / 2)$. Appendix B discusses the effect of the lateral side walls, showing their effect is indeed small for $\delta \ll 1$.

From the velocity scalings given in (2.3), it is clear that the cross-sectional velocity components are much smaller than the axial one. Thus, to the leading order in the two small parameters, the velocity field in this lubrication approximation is dominated by the axial component $V_{Z}$. Terms beyond the leading-order ones can be computed in the standard way, by positing a regular perturbation expansion in the two small parameters $\epsilon$ and $\delta$.

Next, we wish to relate the volumetric flow rate though the channel,

$$
Q:=\int_{-1 / 2}^{+1 / 2} \int_{0}^{H(X, Z)} V_{Z}(X, Y, Z) \mathrm{d} Y \mathrm{~d} X
$$

to the streamwise gradient of the pressure $\mathrm{d} P / \mathrm{d} Z$. Since the flow is steady, $Q$ is constant. Then, substituting $V_{Z}$ from (2.8) into (2.9) and integrating over $Y$, we get (to the leading order in $\delta$ and $\epsilon$ ) the "standard" lubrication theory relation

$$
Q=-\frac{1}{12} \frac{\mathrm{d} P}{\mathrm{~d} Z} \int_{-1 / 2}^{+1 / 2} H(X, Z)^{3} \mathrm{~d} X .
$$

Thus, for a given shape of the top wall of the channel, we can find $Q$ from (2.10). Within the lubrication approximation, the first correction in $\delta$ to 2.10) can be computed as shown in Appendix B. 


\section{Shape of the deformed channel}

In terms of the dimensionless variables from (2.3), the channel shape can thus be expressed as

$$
H(X, Z)=1+\beta U(X, Z),
$$

where we have set $\beta:=u_{\mathrm{c}} / h_{0}$. Here, the dimensionless group $\beta$ controls the compliance of the top wall: for $\beta \ll 1$, the top wall is stiff (equivalently, its deformation is small compared to the undeformed height), and for $\beta \gg 1$ it is soft (equivalently, its deformation is large compared to the undeformed height). Within the lubrication approximation, we do not need to make any assumptions on the smallness of $\beta$ at this stage, however, we expect $\beta>0$ and $\beta \delta \ll 1$ as stated above in $₫ 2$ (see also Appendix B).

If the maximum displacement $\max _{x, z}|u(x, z)|$ of the top wall can be assumed small compared to its thickness $t$, and its thickness $t$ is small compared to its width $w$, then, from the theory of linear elasticity, we know that the steady-state displacement $u(x, z)$ satisfies the Kirchhoff-Love equation for isotropic quasi-static bending of a plate under a transverse load due to the fluid pressure (Love 1888; Timoshenko \& Woinowsky-Krieger 1959; Landau \& Lifshitz 1986):

$$
B \nabla_{\|}^{2} \nabla_{\|}^{2} u=p,
$$

where $B=E t^{3} /\left[12\left(1-\nu^{2}\right)\right]$ is the bending energy (flexural rigidity) of the plate, $E$ is the material's Young's modulus, and $\nu$ is the Poisson ratio of the material; $\nabla_{\|}^{2}$ is the Laplacian operator in the $(x, z)$ coordinates (tangent to the base flow). Equation (3.2) models only bending of the plate, with stretching being assumed negligible; if stretching is judged to be significant, then the Föppl-von Kármán equations can be employed (Timoshenko \& Woinowsky-Krieger 1959; Landau \& Lifshitz 1986).

Using the dimensionless variables from (2.3), equation (3.2) becomes

$$
\delta^{4} \frac{\partial^{4} U}{\partial X^{4}}+2 \epsilon^{2} \delta^{2} \frac{\partial^{4} U}{\partial X^{2} \partial Z^{2}}+\epsilon^{4} \frac{\partial^{4} U}{\partial Z^{4}}=\frac{h_{0}^{4} \Delta p}{B u_{\mathrm{c}}} P .
$$

To obtain a dominant balance in (3.3), we must take $h_{0}^{4} \Delta p /\left(B u_{\mathrm{c}}\right)=\delta^{4}$ or, specifically, this balance sets the characteristic deformation scale to be

$$
u_{\mathrm{c}}=\frac{h_{0}^{4} \Delta p}{B \delta^{4}}=\frac{w^{4} \Delta p}{B} .
$$

Hence, $\beta \equiv u_{\mathrm{c}} / h_{0}=h_{0}^{3} \Delta p /\left(B \delta^{4}\right)$; typical values of $\beta$ are estimated in table 1 below. With $u_{\mathrm{c}}$ determined, equation (3.3) gives, to the leading order,

$$
\frac{\partial^{4} U}{\partial X^{4}}=P(Z)
$$

Physically, this asymptotic limit can be interpreted as follows: Since $w \ll \ell$, the variation of the height of the channel in the streamwise direction occurs over a much longer length scale than the variation of the height in any cross-section. In other words, the deflection of any infinitesimal slice (perpendicular to the flow-wise axial direction) of the top wall does not affect infinitesimal slices nearby. Thus, the Kirchhoff-Love plate theory reduces to the Euler-Bernoulli beam theory for each infinitesimal spanwise slice of the top wall. This limit should be contrasted to the case when $\delta \sim \epsilon$ (and the elastic top wall is not clamped along $X= \pm 1 / 2$ ) in which case bending (and, potentially, tension) in the flow-wise direction dominate (Gomez et al. |2017).

Of course, at this order in the asymptotic analysis, the beam equation (3.5) cannot satisfy the boundary conditions along $Z=0$ or $Z=1$ (the channel's entrance and exit planes). In experiments, the plate is also clamped along $Z=0$ and $Z=1$. From (3.3), it 
is evident that a boundary layer calculation can be done by the rescaling $Z \mapsto(\epsilon / \delta) Z$, which keeps all terms on the left-hand side of (3.3). Thus, any corrections due to clamping at $Z=0$ and $Z=1$ are localized to layers of width $\mathcal{O}(\epsilon / \delta)$, which are a small correction to the system's response in the central part of the channel, given our assumed ordering of small parameters (2.6).

We take the plate to be clamped at $X= \pm 1 / 2$, so (3.5) is subject to the boundary conditions

$$
U( \pm 1 / 2, Z)=0,\left.\quad \frac{\partial U}{\partial X}\right|_{X= \pm 1 / 2}=0 .
$$

Integrating equation (3.5) four times with respect to $X$ and enforcing the boundary conditions from (3.6), we find that

$$
U(X, Z)=\frac{1}{24} P(Z)\left(X+\frac{1}{2}\right)^{2}\left(X-\frac{1}{2}\right)^{2} .
$$

Note that, because $u_{c}$ is set by bending via (3.4), we cannot take a limit as a parameter in (3.7) vanishes and recover the case of a rigid channel (i.e., $U=0$ ).

The top-wall displacement given by (3.7) is a quartic polynomial of the cross-sectional coordinate $X$, which corresponds to our assumption that the system is in a bendingdominated regime. Nevertheless, it should also be noted that a (different) quartic profile is consistent with recent experimental measurements of a similar model system (Ducloué et al. 2017), in which bending and pre-tension can both be considered significant. In other literature (Gervais et al. 2006; Cheung et al. 2012; Ozsun et al. 2013; Raj \& Sen 2016), however, a parabolic (quadratic) profile has often been assumed, even without making a distinction between (or an evaluation of) bending-dominated versus stretching-dominated deformation.

It is often of interest to relate the cross-sectionally averaged displacement to the maximum displacement, since the latter is easier to measure experimentally. From (3.7) we find that

$$
\bar{U}(Z):=\int_{-1 / 2}^{+1 / 2} U(X, Z) \mathrm{d} X=\frac{8}{15} U_{\max }(Z), \quad U_{\max }(Z):=\frac{1}{384} P(Z) .
$$

In passing, we note that the prefactor in $(3.8 \mathrm{~B} a)$, when computed on the basis of the parabolic displacement approximation is $2 / 3$ rather than $8 / 15$; a $25 \%$ difference. The predicted linear scaling of the maximum and cross-sectionally averaged displacements with the pressure is also consistent with estimates of the static deflection of plates by Timoshenko \& Woinowsky-Krieger (1959).

\section{The flow rate-pressure drop relation}

To find the flow rate through the deformed channel, we must evaluate the integral in (2.10) using the top-wall shape stipulated in (3.1):

$$
Q=-\frac{1}{12} \frac{\mathrm{d} P}{\mathrm{~d} Z} \int_{-1 / 2}^{+1 / 2}[1+\beta U(X, Z)]^{3} \mathrm{~d} X .
$$

This integral can be evaluated upon substituting the expression for $U$ from (3.7) to find

$$
Q=-\frac{1}{12} \frac{\mathrm{d} P}{\mathrm{~d} Z}\left[1+\frac{\tilde{\beta}}{10} P(Z)+\frac{\tilde{\beta}^{2}}{210} P(Z)^{2}+\frac{\tilde{\beta}^{3}}{12,012} P(Z)^{3}\right],
$$


where $\tilde{\beta}:=\beta / 24=h_{0}^{3} \Delta p /\left(24 B \delta^{4}\right)$. In passing, we note that (4.2) is a relationship of the form $Q=-\sigma(P) \mathrm{d} P / \mathrm{d} Z$, for some function $\sigma$ that comes about from solving the elasticity problem, as discussed by Rubinow \& Keller (1972).

For a constant imposed flow rate $Q$, (4.2) is a separable first-order ordinary differential equation for the pressure distribution $P(Z)$ subject to $P(1)=0$, whence

$$
Q=\frac{P(Z)}{12(1-Z)}\left[1+\frac{\tilde{\beta}}{20} P(Z)+\frac{\tilde{\beta}^{2}}{630} P(Z)^{2}+\frac{\tilde{\beta}^{3}}{48,048} P(Z)^{3}\right] .
$$

As $Q$ is constant, (4.3) is a polynomial, whose real positive root gives the dimensionless fluid pressure $P$ as a function of the streamwise coordinate $Z$ and the various dimensionless parameters $甘$ For $\tilde{\beta}=0$ (an "infinitely stiff" plate), there is no deformation, and (4.3) reduces to the classical lubrication-theory result for a rectangular channel.

Since the last two terms in the bracket in (4.3) have fairly large denominators, they can be neglected for small as well as moderate values of $\tilde{\beta} P(Z)$, the consequences of which are discussed in Appendix @ Obviously, for larger values of $\tilde{\beta} P(Z)$ these higher-order terms become important.

\subsection{Flow-rate-controlled versus pressure-drop-controlled problems}

For a pressure-driven flow, $\Delta P=1$ under the nondimensionalization $p=\Delta p P$; hence $P(0)=1$. Then, the flow rate-pressure drop relation (4.3) can be evaluated at $Z=0$ to obtain

$$
12 Q=1+\frac{\tilde{\beta}}{20}+\frac{\tilde{\beta}^{2}}{630}+\frac{\tilde{\beta}^{3}}{48,048},
$$

which is a universal dimensionless relationship valid for any applied $\Delta p$.

For a flow-rate controlled situation, $Q=1$ under the nondimensionalization $p=$ $\mu \ell q /\left(h_{0}^{3} w\right) P$; hence $P(0)=\Delta P$. Then, the flow rate-pressure drop relation (4.3) can be evaluated at $Z=0$ to obtain

$$
12=\Delta P\left[1+\frac{\tilde{\beta}}{20} \Delta P+\frac{\tilde{\beta}^{2}}{630}(\Delta P)^{2}+\frac{\tilde{\beta}^{3}}{48,048}(\Delta P)^{3}\right],
$$

which is a universal dimensionless relationship valid for any imposed $q$.

Equations (4.4) and (4.5) can also be interpreted as consistency checks. For a given experiment (or numerical simulation) with an imposed (dimensionless) flow rate $Q$ or an imposed dimensionless pressure drop $\Delta P$, the value of $\tilde{\beta}$ corresponding to the system must satisfy either the (4.4) or (4.5).

\subsection{Comparison with previous models}

Equation (4.3), which contains no fitting parameters, can be compared to equation (10) of Gervais et al. (2006), which incorporates a fitting parameter $\alpha$ and has the dimensional form:

$$
q=\frac{h_{0}^{3} w p(z)}{12 \mu(\ell-z)}\left[1+\frac{3}{2} \frac{\alpha w}{E h_{0}} p(z)+\left(\frac{\alpha w}{E h_{0}}\right)^{2} p(z)^{2}+\frac{1}{4}\left(\frac{\alpha w}{E h_{0}}\right)^{3} p(z)^{3}\right] .
$$

Equation (4.6) was derived by Gervais et al. (2006) under the assumption that the top wall is thick, and the strains within it, $u / w$, are proportional to $p / E$. The proportionality

$\dagger$ Though it is possible to obtain the roots of the quartic polynomial in (4.3) by Ferrari's procedure (see, e.g., Abramowitz \& Stegun 1972, §3.8.3), the explicit formula for the sought positive real root is far too long to be presented here. It can, however, be found easily using Mathematica. 
constant is $\alpha$, and it is unknown a priori. Although Gervais et al. (2006) only discuss the case of a thick top wall that behaves like an elastic half-space, (4.6) has been used by others (see, e.g., Hardy et al. 2009; Cheung et al. 2012; Raj et al. 2017) for microchannels with much thinner top walls that behave as plates. Consequently, when (4.6) is fit to flow pressure-drop data from microchannels with plate-like top walls, a dependence $\alpha \propto$ $(t / w)^{3}$ is observed (Raj et al. 2017).

For the bending-dominated analysis presented herein, we found [similarly to Gervais et al. (2006)] that $u / w \propto p / E$, but on the basis of plate theory. Consequently, it is possible to compare our flow rate-pressure drop relation (4.3) to a generic relation such as (4.6) since they are both based on the top wall displacement scaling with the fluid pressure, i.e., $u / w \propto p / E$. In order to discuss the similarities and differences between these two relations, we note that the dimensional form of (4.3) is

$$
\begin{aligned}
q=\frac{h_{0}^{3} w p(z)}{12 \mu(\ell-z)}\left[1+\frac{1}{480} \frac{w^{4}}{B h_{0}} p(z)+\frac{1}{362,880}\right. & \left(\frac{w^{4}}{B h_{0}}\right)^{2} p(z)^{2} \\
& \left.+\frac{1}{664,215,552}\left(\frac{w^{4}}{B h_{0}}\right)^{3} p(z)^{3}\right] .
\end{aligned}
$$

Clearly, the quantity in the brackets on the right-hand side of (4.6) can be compared with the quantity in the brackets on the right-hand side of (4.7). The caveat here is that we assume that (4.6) can be applied to the bending-dominated top wall deformation regime. In this comparison, then, the role of the parameter $\alpha$ is to absorb the necessary details of the displacement profile and its scaling with various material and geometric constants. For example, the second terms in the brackets of (4.6) and (4.7) can be made to agree if we choose $\alpha=\frac{1}{60}(w / t)^{3}\left(1-\nu^{2}\right)$. We cannot make all terms agree because (4.7) is based on the cross-sectional displacement profile $u(x, z)$ derived in 93 , while Gervais et al. (2006) postulated that $u / w=\alpha p / E$.

Raj \& Sen (2016) and Raj et al. (2017) also observed that $\alpha$ need not be a fitting parameter but, instead, must be related to the various geometric and elasticity constants involved in the problem. A difference between the approaches of Raj \& Sen (2016) and Raj et al. (2017) and ours is that their derivations are based on correlating the maximum of the cross-sectionally-averaged displacement to the pressure via various approximations/assumptions. In particular, Raj \& Sen (2016) make the assumption of a parabolic top wall deflection, which does not appear to be well-substantiated by their measurements. Consequently, the (dimensional) "compliance parameters," $f_{\mathrm{p}}$ introduced by Raj \& Sen (2016) and $\Omega$ introduced by Raj et al. (2017), are derived differently from (the dimensionless) $\tilde{\beta}$ introduced above on the basis of the perturbation expansion of the coupled fluid-structure interaction problem.

More importantly, however, Rai \& Sen (2016) based their analysis on the shell-stretching correlation $u / w \propto(p / E)^{1 / 3}$, which yields a model that cannot be compared to those based on linearly proportional displacement and pressure (such as those discussed above). On the other hand, the model flow rate-pressure drop relation of Raj et al. (2017) was based on a plate-bending correlation, so it can be directly compared to (4.7). Unfortunately, there are misprints in the derivation of equation (12) of Raj et al. (2017), i.e., the model flow rate-pressure drop relation therein. Specifically, the compliance parameter $\Omega$ as given in equation (10) of Raj et al. (2017) should read $\Omega=\frac{1}{99} E^{-1}\left(w / h_{0}\right)(w / t)^{3}\left(1-\nu^{2}\right)$ and the flow rate-pressure drop relation from equation (12) of Raj et al. (2017) should 
(a)

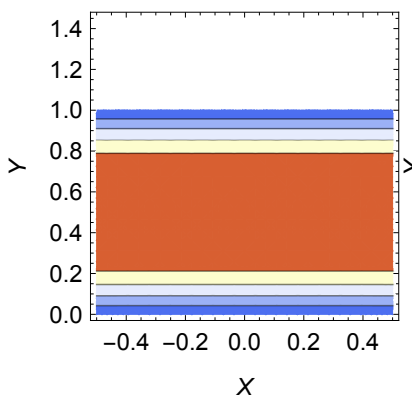

(b)

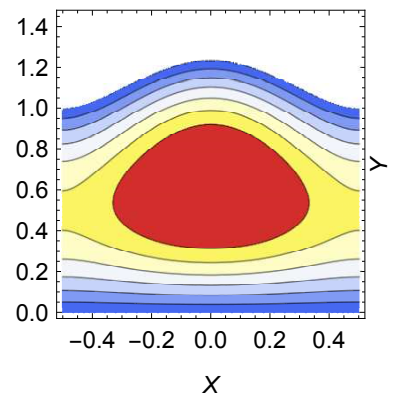

(c)

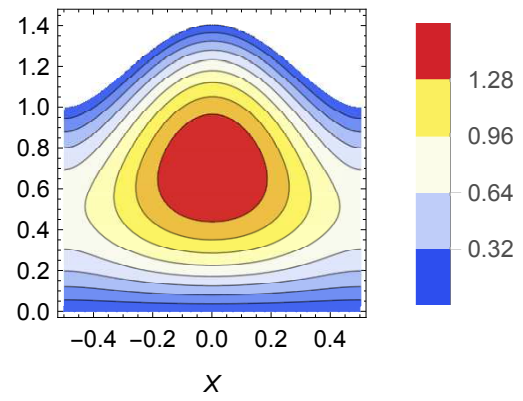

Figure 2: Contours of the axial velocity, $V_{Z}$, as a function of the $(X, Y)$ coordinates in the $Z=0.25$ cross-section of the channel with $Q=1$ and for different $\tilde{\beta}$ values, computed from (2.8) with the pressure given by the appropriate real root of (4.3) and the channel height given by (3.1) and (3.7). (a) Solid top wall $(\tilde{\beta}=0)$. (b) Elastic top wall with $\tilde{\beta}=0.5$. (c) Elastic top wall with $\tilde{\beta}=1$.

read

$$
q=\frac{h_{0}^{3} w p(z)}{12 \mu(\ell-z)}\left[1+\frac{3}{2} \Omega p(z)+\Omega^{2} p(z)^{2}+\frac{1}{4} \Omega^{3} p(z)^{3}\right] .
$$

On comparing (4.8) to (4.6), it follows that $\alpha=\frac{1}{99}(w / t)^{3}\left(1-\nu^{2}\right)$. Unsurprisingly, this comparison yields a constant $\alpha$, which is no longer a fitting parameter. The latter prediction differs from ours above in the prefactor $\frac{1}{99} \approx 0.011$ versus $\frac{1}{60} \approx 0.017$. Furthermore, as was the case with (4.6), (4.8) cannot be made to agree with (4.7) because (4.8) was derived following the approach of Gervais et al. (2006), while (4.7) was derived using the actual cross-sectional displacement profile $u(x, z)$.

\section{Illustrated examples and comparison with experiments}

First, consider the leading-order axial velocity component (2.8). Clearly, because $P=$ $P(Z)$ and $H=H(X, Z)$, the deformation of the channel's top wall introduces dependence upon both of the coordinates (i.e., $X$ and $Z$ ) that are not present in the leading-order velocity in a rigid channel, namely $V_{Z}(X, Y, Z)=6 Q(1-Y) Y$. The effect of the top wall's elasticity on the axial velocity is illustrated in figure 2. Specifically, it is evident that the cross-sectional shape's deformation introduces variability in the $X$-direction, which leads to faster flow near the channel's centerline (compared to the rest of the cross-section), consistent with the numerical simulations shown in figure 3 of (Gervais et al. 2006).

Second, the channel's top wall deformation is illustrated in figure 3. In dimensionless variables, figure 3( $a$ ) shows a surface plot of the top-wall displacement as a function of the spatial coordinates, while figure $3(b)$ shows the maximum displacement of the top wall as a function of the flow rate $Q$. The shapes of the latter curves agree qualitatively with those in figures 5 and 7 of Gervais et al. (2006), respectively.

Third, the nonlinear flow rate-pressure drop curves for a deformable channel are illustrated in figure 4 $(a)$ for different values of $\tilde{\beta}$. Their shapes agree qualitatively with Gervais et al. (2006) (figure 9 therein). From figure 4(a), it is clear that, for $\tilde{\beta} \neq 0$ (a deformable channel), the value of $Q$ for a given $\Delta P$ can be significantly larger as $\beta$ is increased, i.e., for softer channels. Meanwhile, figure $4(b)$ shows the pressure in the microchannel as a function of the axial coordinate using the implicit expression (4.3). 

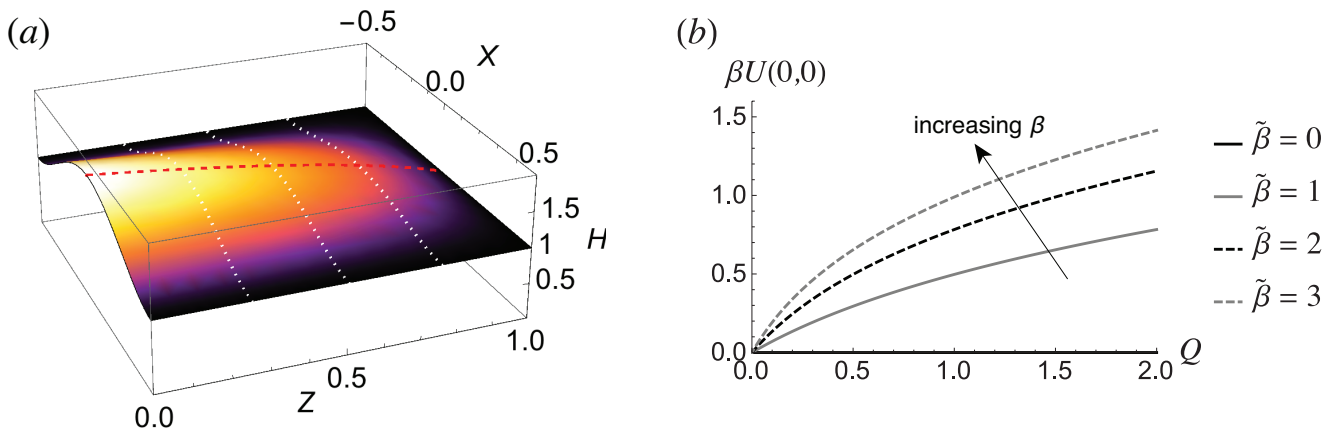

Figure 3: (a) $H(X, Z)$ computed from (3.7) with $P(Z)$ computed from (4.3), for $\tilde{\beta}=2$ and $Q=1$; thick dashed line represents $1+\beta U_{\max }(Z)$, thin dotted lines are a guide to the eye. (b) Maximum displacement, i.e., $H(0,0)-1=\beta U(0,0)$ calculated from (3.7) with $P(Z)$ computed from (4.3), as function of the flow rate $Q$ and different values of $\tilde{\beta}$.

(a) $\Delta P$

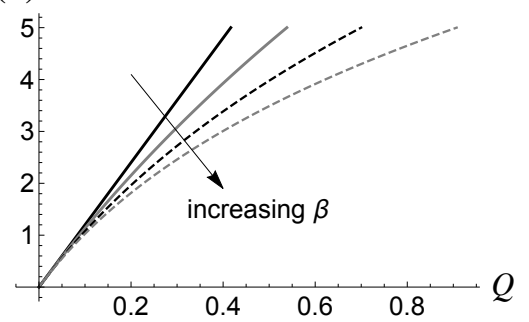

(b) $P(Z)$

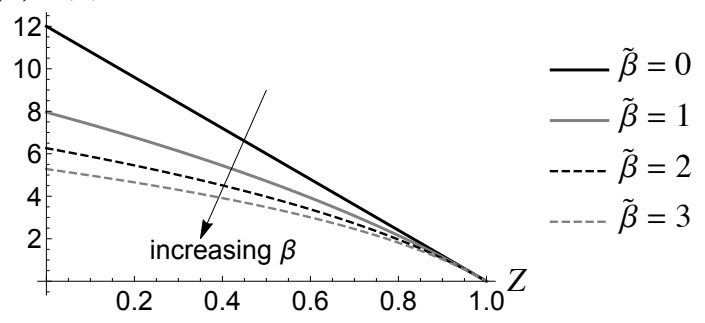

Figure 4: (a) Pressure drop across the channel, $\Delta P=P(0)$, computed via (4.3), as a function of the flow rate $Q$ for different values of $\beta$. (b) Pressure $P$ as a function of the axial coordinate $Z$ computed by inverting (4.3); $Q=1$.

Clearly, a contribution of flow-elasticity coupling is to make the pressure distribution in the channel nonlinear. Furthermore, since the channel cross-sectional area increases due to the top wall's deformation, a smaller pressure gradient is needed to achieve the same volumetric flow rate.

Next, we present comparisons between the predictions of our theoretical analysis and experimental results previously published in the literature. To this end, table 1 summarizes the values of the physical parameters in the flow-rate-controlled PDMS microchannels experiment, which best matches the assumptions of our theoretical developments, labeled "S4" in (Ozsun et al. 2013). The ranges of $\beta$ and $\tilde{\beta}$, which were computed using (4.4), correspond to the range of flow rates considered by Ozsun et al. (2013), namely $q=10$ to $50 \mathrm{~mL} / \mathrm{min}$, or $Q=0.1$ to 0.15 . From table 1 , which also shows the values of the dimensionless quantities that we have introduced, we see that $\delta \approx 0.144$ while $\epsilon \approx 0.02$, which satisfies our assumed asymptotic ordering: $0<\epsilon \ll \delta \ll 1$. Regarding our assumption that plate-bending theory captures the mechanics of the top-wall deformation, we note, from table 1, that $t / w=0.11 \ll 1$, while $\max _{x, z}|u(x, z)| / t \leqslant 0.43<1$ based on the results of Ozsun et al. (2013) (with 0.43 being the value at the largest flow rate considered therein).

To make a quantitative comparison between theory and experiment, figure 5 shows a 


\begin{tabular}{|c|c|c|c|c|c|c|c|c|c|c|c|}
\hline $\begin{array}{l}h_{0} \\
(\mathrm{~mm})\end{array}$ & $\begin{array}{l}w \\
(\mathrm{~mm})\end{array}$ & $\begin{array}{l}\ell \\
(\mathrm{mm})\end{array}$ & $\begin{array}{l}\Delta p \\
(\mathrm{kPa})\end{array}$ & $\begin{array}{l}t \\
(\mathrm{~mm})\end{array}$ & $\begin{array}{l}E \\
(\mathrm{MPa})\end{array}$ & $\nu$ & $\begin{array}{l}B \\
(\mu \mathrm{J})\end{array}$ & $\delta$ & $\epsilon$ & $\beta$ & $\tilde{\beta}$ \\
\hline 0.244 & 1.7 & 15.5 & $1-5$ & 0.2 & $\approx 1.6$ & 0.499 & $\approx 1.60$ & 0.144 & 0.0244 & $24.5-97$ & $1-4$ \\
\hline
\end{tabular}

Table 1: Values of the physical parameters for the flow-rate-controlled PDMS microchannels experimental system S4 of Ozsun et al. (2013), which most closely matches the assumptions of our theoretical developments.

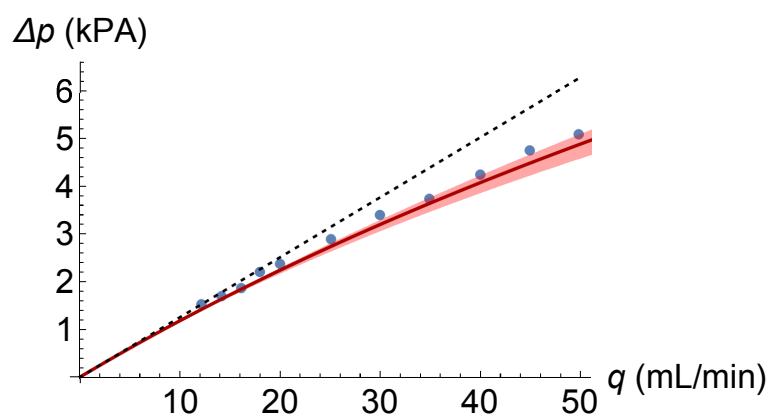

Figure 5: Dimensional flow rate-pressure drop relationship: comparison between our asymptotic theory (solid curve) [i.e., (4.7) evaluated at $z=0$ ], including a shaded trust region based on taking $E=1.6 \times 10^{6} \pm 25 \% \mathrm{MPa}$, and the experimental data from the S4 system of Ozsun et al. (2013) (symbols) with error bars smaller than the symbols according to Ozsun et al. (2013). The dotted line represents the standard lubrication-theory linear flow rate-pressure drop relation for a rigid rectangular channel, i.e., $\Delta p=12 \mu \ell q /\left(h_{0}^{3} w\right)$.

plot of the dimensional flow rate-pressure drop relation (4.7) based on our asymptotic theory, the corresponding experimental measurements from the $\mathrm{S} 4$ system of Ozsun et al. (2013) and a reference line corresponding to the lubrication-theory result for a solid rectangular channel. Clearly, there is good agreement between theory and experiment, especially with respect to the shape of the flow rate-pressure drop curve. In particular, the crossover from a linear (at low $q$ ) to a nonlinear (at high $q$ ) regime can be observed in the plot.

The theoretical curve shown in figure 5 systematically underpredicts the pressure drop by about $5 \%$. We attribute this small discrepancy to two factors. First, the deformation of the S4 microchannel is not insignificant: as reported in table 1 of Ozsun et al. (2013), $\max _{x, z}|u(x, z)| / t \approx 0.43$. Such a maximum displacement, given the top wall's thickness, might be considered at the edge of applicability of the Kirchhoff-Love (linearly elastic) plate theory. Hence, the agreement might be improved if a different top-wall elasticity model, which accounts for the nontrivial wall thickness, were used. Second, the elasticity parameters of the PDMS microchannels are not well characterized by Ozsun et al. (2013). We have estimated a value of $E \approx 1.6 \mathrm{MPa}$ using their description of the experimental procedure, cross-referencing against the data on PDMS material properties tabulated by Johnston et al. (2014) and treating the hydrostatic loading data (Ozsun et al. 2013, figure $1(e, f)$ ) as a "bulge test" (Small \& Nix 1992). However, there is inherent uncertainty in such an estimate, which is why we have added a confidence region (corresponding to a $\pm 25 \%$ variation in the Young's modulus $E$ ) around the theoretical curve in fig. 5 . 
This uncertainty appears to capture most of the (small) discrepancy between theory and experiment. Therefore, it is clear that figure 5 shows that a quantitative prediction of the flow rate-pressure drop curve for a long shallow microchannel is possible without fitting parameters.

Finally, based on the discussion 4.2 it might be appropriate to think of the fitting parameter $\alpha$ of Gervais et al. (2006) as constant given by $\alpha=\frac{1}{60}(w / t)^{3}\left(1-\nu^{2}\right)$. Then, for the S4 experiments of Ozsun et al. (2013), we find that fitting the data to (4.6) evaluated at $z=0$ yields $\alpha \approx 5.91-$ Meanwhile, $\alpha=\frac{1}{60}(w / t)^{3}\left(1-\nu^{2}\right) \approx 7.68$ using the values given in table 1

\section{Discussion and conclusions}

Using asymptotic techniques, we have derived a flow rate-pressure drop relation for low-Reynolds-number flow in a shallow deformable channel. Our result, which contains no fitting parameters, can be applied to the design and construction of soft microfluidic devices, where the flow and the elastic deformation of the domain are coupled. Specifically, we treated the case of bending-dominated deformation, which is governed by the plate equation (3.2). This restricts our analysis to microchannels for which the maximum deformation $u_{\max }:=\max _{x, z}|u(x, z)|$ is much smaller than the thickness of the top wall, which is, in turn, much smaller than the channel's width: $u_{\max } \ll t \ll w$.

To make clear the limitations of our model, consider first the case when $t \gg w$, i.e., the top wall is much thicker than the width of the channel, as is the case in the experiments of Gervais et al. (2006). Then, the top wall behaves like an elastic half-space rather than a plate. An extension of the present work would require us to show a similar decoupling between the flow-wise and transverse deformation, as was done in $\$ 3$ perhaps adapting the solution for a uniform load distributed over a finite width of an elastic halfspace Johnson 1985, §2.4). Now, consider the case when $u_{\max } \gg t$, i.e., the top wall's deformation is much larger than its thickness, as is the case in some of the experiments of Ozsun et al. (2013). Now, the top wall behaves like a membrane rather than a plate. Once again, an extension of the present work would be to revisit $\sqrt{3}$ starting from a model of stretching-dominated deformation, perhaps adapting the approximate solution from (Timoshenko \& Woinowsky-Krieger 1959, Art. 101). Each case would require a careful recalculation of the flow rate-pressure drop relation and appropriate comparisons against applicable experiments. Unfortunately, at this time, few reliable measurements of the topwall displacement of microchannels under hydrodynamic conditions have been published.

Returning to our results for the bending-dominated case, in dimensional variables and keeping only the leading-order elasticity contribution in (4.7), we can summarize the key result:

$$
q \approx \frac{h_{0}^{3} w \Delta p}{12 \mu \ell}\left[1+\frac{3}{160}\left(\frac{w}{t}\right)^{3}\left(\frac{w}{h_{0}}\right)\left(\frac{\Delta p}{E}\right)\right],
$$

where $q$ is the flow rate, $\Delta p$ is the pressure drop, $w$ is the channel width, $h_{0}$ is the undeformed channel height, $\ell$ is the channel length, $t$ is the top wall's thickness, and $E$ is the Young's modulus of the material (assuming an incompressible material, i.e., a Poisson ratio $\nu=1 / 2$ ). In particular, (6.1) highlights the strong dependence on the plate geometry through the factor of $(w / t)^{3}$, which is assumed $\gg 1$ in our plate-bending model.

$\dagger$ Here, we have used MATHEMATiCA's built-in nonlinear least-squares subroutine FindFit. Note, however, that the best-fit value is sensitive to small errors introduced in digitizing the data from (Ozsun et al. 2013). 
Note that the ratio outside the square brackets in (6.1) represents the leading-order term (in $h_{0} / w \equiv \delta$ ) in the flow rate-pressure drop relation for a shallow rectangular channel.

However, the boundary conditions in the transverse $x$-direction are not enforced in this approach. This defect comes from the shallowness assumption that allows us to neglect $\mathcal{O}\left(\delta^{2}\right)$ terms in the asymptotic expansion. A potential remedy is to relax the assumption that $\delta \ll 1$ and solve the full-2D leading-order flow problem using the domain perturbation technique (Van Dyke 1975; Lebovitz 1982). An alternative approach, consistent with lubrication theory, is presented in Appendix B. Finally, future work might include computing the remaining two components of the leading-order velocity field. This threedimensional case requires further consideration because, as shown by Lauga et al. (2004), if the cross-section is varying in the flow-wise direction, then the flow cannot be planar, so the continuity equation alone does not yield the remaining velocity component as in standard lubrication theory. It would also be of interest to compute higher-order perturbative corrections to the lubrication theory velocity profile given in $\$ 2$ following, e.g., Tavakol et al. (2017).

\section{Acknowledgements}

This work was initiated while I.C.C. and V.C. were working with H.A.S. at Princeton University. At that time, I.C.C. was supported by the National Science Foundation (NSF) under grant No. DMS-1104047. H.A.S. thanks the NSF for support via grant No. CBET-1132835. We thank the anonymous referees for their incisive comments, questions and suggestions, which helped improve the manuscript. Specifically, one of the referee's remarks lead to the calculation in Appendix B

\section{Appendix A. Explicit pressure and velocity expressions for a stiff top wall}

Note that the last two terms in the bracket on the right-hand side of (4.3) have large denominators. Thus, let us neglect these two terms under the assumption that $\max _{0 \leqslant Z \leqslant 1} \tilde{\beta} P(Z) \equiv \tilde{\beta} P(0)=\mathcal{O}(1)$. This approximation reduces the flow rate-pressure drop relation to a quadratic equation that can be solved explicitly for $P$ :

$$
P(Z)=-\frac{10}{\tilde{\beta}}\left[1 \pm \sqrt{1+\frac{12}{5} \tilde{\beta} Q(1-Z)}\right],
$$

where we must pick the "-" sign above because our convention is that $P(1)=0$. The approximate expression (A1) highlights the fact that $P$ is a nonlinear function of $Z$ in a deformable channel, unlike the rigid-channel relation $P(Z)=12(1-Z)$, which is linear. The maximum error committed by (A 1) is for $P(Z=0)$, which corresponds to the error in the full pressure drop $\Delta P=P(0)$. Figure 6 shows the percent error in $\Delta P$ as predicted by (A 1) compared to the result from (4.5) in a flow-rate controlled situation $(Q=1)$. It is evident that the error is modest $(\lesssim 15 \%)$ for up to $\tilde{\beta}=2$.

Next, we expand (A1) into a Taylor series (for $\tilde{\beta} Q \ll 1$ ) to highlight the first perturbative correction to the rigid-channel expression:

$$
P(Z)=12 Q(1-Z)\left[1-\frac{3}{5} \tilde{\beta} Q(1-Z)+\mathcal{O}\left(\tilde{\beta}^{2} Q^{2}\right)\right] .
$$




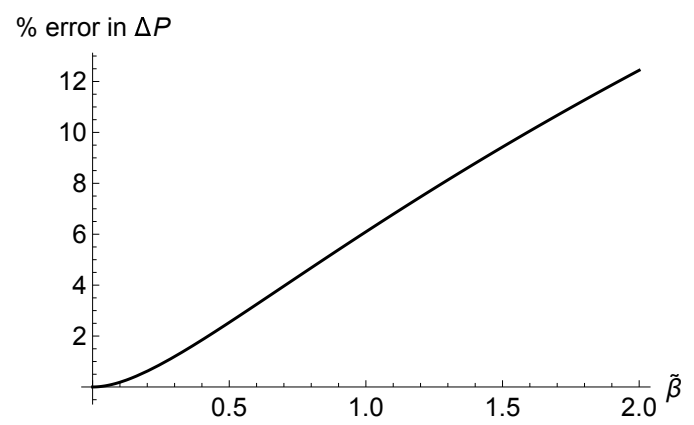

Figure 6: Percent error in the full pressure drop $\Delta P=P(0)$ as calculated via the approximate explicit relation (A 1), for a flow-rate controlled situation $(Q=1)$, and compared to pressure drop from the "full" implicit relation (4.5), as a function of the compliance parameter $\tilde{\beta}$.

Now, differentiating $P$ with respect to $Z$ from either (A 1) or (A 2), we find

$$
-\frac{\mathrm{d} P}{\mathrm{~d} Z}=12 Q\left[1+\frac{12}{5} \tilde{\beta} Q(1-Z)\right]^{-1 / 2}=12 Q\left[1-\frac{6}{5} \tilde{\beta} Q(1-Z)+\mathcal{O}\left(\tilde{\beta}^{2} Q^{2}\right)\right] .
$$

Substituting $-\mathrm{d} P / \mathrm{d} Z$ from (A 3) into (2.8), and using (3.1) with $U$ given by (3.7), we obtain the velocity distribution

$$
\begin{aligned}
V_{Z}(X, Y, Z)= & 6 Q\left[1+\frac{12}{5} \tilde{\beta} Q(1-Z)\right]^{-1 / 2} \\
& \times\left\{1-10\left[1-\sqrt{1+\frac{12}{5} \tilde{\beta} Q(1-Z)}\right]\left(X+\frac{1}{2}\right)^{2}\left(X-\frac{1}{2}\right)^{2}-Y\right\} Y .
\end{aligned}
$$

Now, we expand (A4 4 into a Taylor series (for $\tilde{\beta} Q \ll 1$ ) to highlight the first perturbative correction to the rigid-channel expression:

$$
V_{Z}(X, Y, Z)=6 Q(1-Y) Y+\frac{9}{10} \tilde{\beta} Q^{2}\left(80 X^{4}-40 X^{2}+8 Y-3\right) Y(1-Z)+\mathcal{O}\left(\tilde{\beta}^{2} Q^{2}\right) .
$$

Clearly, the first correction to the velocity field, unlike the corresponding one to the pressure in (A2), introduces dependence upon both of the coordinates (i.e., $X$ and $Z$ ) that are not present in the leading-order term.

Equation (A4) provides a tractable expression from which to compute the maximum velocity for a given $Z$ cross-section with $Q$ and $\tilde{\beta}$ as parameters:

$$
V_{Z, \max }(Z ; Q, \tilde{\beta}):=\max _{\substack{-1 / 2<X<1 / 2 \\ 0<Y<H(X, Z)}} V_{Z}(X, Y, Z) .
$$

Performing this standard calculation using the approximate velocity expression (A 4) yields

$$
V_{Z, \max }(Z ; Q, \tilde{\beta}) \simeq \frac{3}{2} Q\left[1+\frac{3}{10} \tilde{\beta} Q(1-Z)\right] \quad(\tilde{\beta} Q \ll 1) .
$$

To the leading order in $\tilde{\beta} Q$, equation (A 7) predicts that the dependence upon $Z$ vanishes at the downstream end of the microchannel, which is consistent with the fact that there is no deformation of the top wall there. 


\section{Appendix B. Effect of the lateral side walls}

The effect of the lateral side walls becomes important if the channel is not necessarily shallow, i.e., $\delta \equiv h_{0} / w \nless$. Returning to the fluid's governing equations (2.4)-(2.5), we can retain terms involving $\delta$, while still only considering the equations at the leading order in $\epsilon \equiv h_{0} / \ell \ll 1$. In other words, the Stokes equations (2.5) reduce to

$$
\delta^{2} \frac{\partial^{2} V_{Z}}{\partial X^{2}}+\frac{\partial^{2} V_{Z}}{\partial Y^{2}}=\frac{\partial P}{\partial Z}, \quad 0=\frac{\partial P}{\partial Y}, \quad 0=\frac{\partial P}{\partial X}, \quad(B 1 a, b, c)
$$

and the continuity equation (2.4) remains unchanged. Clearly, calculating the flow ratepressure drop relation for channels that are not necessarily shallow requires solving a two-dimensional (2D) problem since the flow profile depends on both the $X$ and $Y$ crosssectional coordinates. Unfortunately, the problem is posed on a non-rectangular domain: $\{(X, Y) \mid-1 / 2 \leqslant X \leqslant+1 / 2,0 \leqslant Y \leqslant H(X, Z)\}$, where $H(X, Z) \equiv 1+\beta U(X, Z)$ with the displacement $U(X, Z)$ computed self-consistently through (3.5), which remains unchanged even if $\delta=\mathcal{O}(1)$. The no-slip condition provides boundary conditions at the walls: $V_{Z}( \pm 1 / 2, Y)=V_{Z}(X, 0)=V_{Z}(X, H(X, Z))=0$.

Although the difficulty of solving a $2 \mathrm{D}$ problem on a non-rectangular domain can be overcome by the domain perturbation technique (Van Dyke 1975; Lebovitz 1982), this approach would require that we expand the channel's shape in a power series in $\beta \ll 1$ as well (i.e. a nearly rectangular domain). However, $\beta \nless 1$ for this problem, as discussed above, for any $\delta$ including $\delta=\mathcal{O}(1)$. Instead, we proceed by a lubrication argument in which $\beta$ can be large (how large will be specified below) but $\delta \ll 1$.

To this end, first we solve (B1) on the rectangular domain $(X, Y) \in[-1 / 2,+1 / 2] \times$ $[0,1]$. This problem has a known solution, which can be found by separation of variables (e.g., Bruus 2008, §3.4.6):

$$
\begin{aligned}
V_{Z}(X, Y)=-\frac{\mathrm{d} P}{\mathrm{~d} Z}\{ & \frac{1}{2}(1-Y) Y \\
& \left.-\frac{4}{\pi^{3}} \sum_{n=1}^{\infty} \frac{1}{(2 n-1)^{3}} \frac{\cosh [(2 n-1) \pi X / \delta]}{\cosh [(2 n-1) \pi /(2 \delta)]} \sin [(2 n-1) \pi Y]\right\},
\end{aligned}
$$

hence,

$$
Q=-\frac{1}{12} \frac{\mathrm{d} P}{\mathrm{~d} Z}[1-\kappa(\delta)], \quad \kappa(\delta):=\sum_{n=1}^{\infty} \frac{1}{(2 n-1)^{5}} \frac{192}{\pi^{5}} \delta \tanh \left[\frac{(2 n-1) \pi}{2 \delta}\right]
$$

for a channel of fixed rectangular cross-section with aspect ratio $\delta=h_{0} / w$. Furthermore, for future reference, we note the approximation $\kappa(\delta) \approx \kappa_{0} \delta$ as $\delta \rightarrow 0^{+}$, where $\kappa_{0}=\left(192 / \pi^{5}\right)(31 / 32) \zeta(5) \approx 0.630$, and $\zeta(\xi) \equiv \sum_{n=1}^{\infty} n^{-\xi}$ is the Riemann zeta function (Abramowitz \& Stegun 1972, §23.2).

For $\delta \ll 1$, the terms in the summation over $n$ in $(\mathrm{B} 2$ lead to small corrections that are localized in a boundary layer near the side walls $X= \pm 1 / 2$. [Of course, from (2.5c), it is evident that a boundary-layer calculation can be done by the rescaling $X \mapsto \delta X$, which keeps the first term on the left-hand side of (2.5c), to arrive precisely at (B 2) for a rigid channel.] The net effect, which is captured in ( $\overline{\mathrm{B} 3})$, is that the flow rate is reduced by $\kappa(\delta)$ due to friction at the $X= \pm 1 / 2$ sidewalls. Moreover, since the top wall is assumed to be clamped and, thus, $H(X, Z) \rightarrow 1$ as $X \rightarrow \pm 1 / 2$, then the latter observation is true even for a shape-deformed channel in which $Y \in[0, H(X, Z)]$.

Thus, within the lubrication approximation, if we wish to estimate the leading-order contribution of drag due to the sidewalls at $X= \pm 1 / 2$ even in the case of a deformable 
cross-section, then we can employ (B3), provided that we "rescale" it to the domain $\{(X, Y) \mid-1 / 2 \leqslant X \leqslant+1 / 2,0 \leqslant Y \leqslant H(X, Z)\}$ :

$$
\begin{aligned}
V_{Z}(X, Y, Z) & =-\frac{\mathrm{d} P}{\mathrm{~d} Z}\left\{\frac{1}{2}[H(X, Z)-Y] Y\right. \\
& \left.-\frac{4}{\pi^{3}} \sum_{n=1}^{\infty} \frac{1}{(2 n-1)^{3}} \frac{\cosh [(2 n-1) \pi X / \delta]}{\cosh [(2 n-1) \pi /(2 \delta)]} \sin \left[(2 n-1) \pi \frac{Y}{H(X, Z)}\right]\right\} .
\end{aligned}
$$

Why should (B 4) apply? Obviously, in the limit as $\delta \rightarrow 0^{+}$, we obtain the result in 92 For $\delta \neq 0$, we must now consider the summation term. If $\delta \ll 1$, then the summation term in (B4 $)$ is localized in boundary layers near $X= \pm 1 / 2$, independently of $Y$. It is easy to verify that (B4) satisfies all the boundary conditions, namely $V_{Z}( \pm 1 / 2, Y)=V_{Z}(X, 0)=$ $V_{Z}(X, H(X, Z))=0$. However, the velocity profile (B 4) does not satisfy the governing equation (B 1). The error committed is $\mathcal{O}(\beta \delta)$. Therefore, if $\delta \ll 1(\Rightarrow 1 / \delta \gg 1)$, then (B 4) is asymptotically valid for $\beta$ as large as $\beta=\mathcal{O}(1 / \delta)$; in other words, (B 4) applies for $\beta \ll 1 / \delta$, or $u_{\mathrm{c}} \ll w / \delta^{2}$ (i.e., the deformation does not necessarily have to be small).

Substituting (B4) into (2.9), we find the flow rate:

$$
\begin{aligned}
Q=-\frac{1}{12} \frac{\mathrm{d} P}{\mathrm{~d} Z}[1+ & \frac{\tilde{\beta}}{10} P(Z)+\frac{\tilde{\beta}^{2}}{210} P(Z)^{2}+\frac{\tilde{\beta}^{3}}{12,012} P(Z)^{3} \\
& \left.-\frac{96}{\pi^{4}} \sum_{n=1}^{\infty} \frac{1}{(2 n-1)^{4}} \int_{-1 / 2}^{+1 / 2} \frac{\cosh [(2 n-1) \pi X / \delta]}{\cosh [(2 n-1) \pi /(2 \delta)]} H(X, Z) \mathrm{d} X\right] .
\end{aligned}
$$

The last integral can be evaluated exactly based on (3.7), and it gives the correction to (4.2) due to the lateral side walls at $X= \pm 1 / 2$. For the purposes of finding the leading-order correction (in $\delta \ll 1$ ), it suffices to note that

$$
\int_{-1 / 2}^{+1 / 2} \frac{\cosh [(2 n-1) \pi X / \delta]}{\cosh [(2 n-1) \pi /(2 \delta)]} H(X, Z) \mathrm{d} X=\frac{2}{(2 n-1) \pi} \delta \tanh \left[\frac{(2 n-1) \pi}{2 \delta}\right]+\mathcal{O}\left(\delta^{3}\right)
$$

Thus, (4.3) corrected to the leading-order contribution due to drag at the sidewalls at $X= \pm 1 / 2$ in the case of a deformable cross-section, is found by integrating (B 5) with respect to $Z$ subject to $P(1)=0$ and neglecting terms of $\mathcal{O}\left(\delta^{3}\right)$ :

$$
Q=\frac{P(Z)}{12(1-Z)}\left[1+\frac{\tilde{\beta}}{20} P(Z)+\frac{\tilde{\beta}^{2}}{630} P(Z)^{2}+\frac{\tilde{\beta}^{3}}{48,048} P(Z)^{3}-\kappa_{0} \delta\right]
$$

The approach we have outlined in this appendix should be contrasted to how the effect of sidewalls has been treated by Cheung et al. (2012) and the literature derived thereof (e.g., Rai \& Sen 2016; Rai et al. 2017). The approach based on (Cheung et al. 2012) is an extension of the Gervais et al. (2006) model to channels that are not necessarily shallow, meaning it does not account for the details of the deformation profile in the cross-section. Cheung et al. (2012) include the factor $[1-\kappa(\delta)] \approx\left[1-\kappa_{0} \delta\right]$ from (B3) in the integral defining the flow rate, and then account for the variable channel height by replacing it with $\left[1-\kappa_{0} \delta H(X, Z)\right]$. This translates into our analysis as multiplying the integrand of (2.10) by $\left[1-\kappa_{0} \delta H(X, Z)\right]$. Using the expression for the channel shape 
given in (3.1), we obtain

$$
Q=-\frac{1}{12} \frac{\mathrm{d} P}{\mathrm{~d} Z}\left\{\int_{-1 / 2}^{+1 / 2}[1+\beta U(X, Z)]^{3}\left(1-\kappa_{0} \delta[1+\beta U(X, Z)]\right) \mathrm{d} X\right\} .
$$

Then, substituting the expression for $U(X, Z)$ from (3.7) into (B 8) and carrying out the integrations over $X$ and $Z$, we find

$$
\begin{aligned}
Q= & \frac{P(Z)}{12(1-Z)}\left\{1+\frac{\tilde{\beta}}{20} P(Z)+\frac{\tilde{\beta}^{2}}{630} P(Z)^{2}+\frac{\tilde{\beta}^{3}}{48,048} P(Z)^{3}\right. \\
& \left.-\kappa_{0} \delta\left[1+\frac{\tilde{\beta}}{15} P(Z)+\frac{\tilde{\beta}^{2}}{315} P(Z)^{2}+\frac{\tilde{\beta}^{3}}{12,012} P(Z)^{3}+\frac{\tilde{\beta}^{4}}{1,093,950} P(Z)^{4}\right]\right\} .
\end{aligned}
$$

Equation (B9) could be compared to equation (10) of Cheung et al. (2012). However, this approximation has the effect of overestimating the volumetric flow rate loss due drag at the sidewalls, as the factor $\left[1-\kappa_{0} \delta H(X, Z)\right]$ now modifies the flux even at the center of the channel, which in turn introduces the four terms depending on $P(Z)$ in the brackets in (B9).

In summary, we have shown via a perturbation expansion in this Appendix that, to leading order in $\delta \ll 1$, equation (B7), rather than (B9), contains the asymptotic correction to the flow rate.

\section{REFERENCES}

Abramowitz, M. \& Stegun, I. A., ed. 1972 Handbook of Mathematical Functions with Formulas, Graphs, and Mathematical Tables, Applied Mathematics Series, vol. 55. Washington, D.C.: National Bureau of Standards, http://www.nr.com/aands/

Ando, K., Sanada, T., Inaba, K., Damazo, J. S., Shepherd, J. E., Colonius, T. \& Brennen, C. E. 2011 Shock propagation through a bubbly liquid in a deformable tube. $J$. Fluid Mech. 671, 339-363.

Anoop, R. \& Sen, A. K. 2015 Capillary flow enhancement in rectangular polymer microchannels with a deformable wall. Phys. Rev. E 92, 013024.

Bahga, S. S., Bercovici, M. \& Santiago, J. G. 2012 Robust and high-resolution simulations of nonlinear electrokinetic processes invariable cross-section channels. Electrophoresis 33, 3036-3051.

Bodnár, T., Galdi, G. P. \& S̆. NEČASOvÁ, ed. 2014 Fluid-Structure Interaction and Biomedical Applications. Advances in Mathematical Fluid Mechanics . Basel: Birkhäuser.

Bruus, H. 2008 Theoretical Microfluidics. Oxford: Oxford University Press.

Chakraborty, D., Prakash, J. R., Friend, J. \& Yeo, L. 2012 Fluid-structure interaction in deformable microchannels. Phys. Fluids 24, 102002.

Cheung, P., Toda-Peters, K. \& Shen, A. Q. 2012 In situ pressure measurement within deformable rectangular polydimethylsiloxane microfluidic devices. Biomicrofluidics 6, 026501.

CONRAD, W. A. 1969 Pressure-flow relationships in collapsible tubes. IEEE Trans. Biomedical Eng. BME-16, 284-295.

Dendukuri, D., Gu, S. S., Pregibon, D. C., Hatton, T. A. \& Doyle, P. S. 2007 Stop-flow lithography in a microfluidic device. Lab Chip 7, 818-828.

Ducloué, L., Hazel, A. L., Thompson, A. B. \& Juel, A. 2017 Reopening modes of a collapsed elasto-rigid channel. J. Fluid Mech. 819, 121-146.

Duprat, C. \& Stone, H. A. 2016 Fluid-Structure Interactions in Low-Reynolds-Number Flows. Cambridge, UK: The Royal Society of Chemistry.

Elbaz, S. B. \& GAT, A. D. 2014 Dynamics of viscous liquid within a closed elastic cylinder subject to external forces with application to soft robotics. J. Fluid Mech. 758, 221-237.

Elbaz, S. B. \& Gat, A. D. 2016 Axial creeping flow in the gap between a rigid cylinder and a concentric elastic tube. J. Fluid Mech. 806, 580-602. 
Fung, Y. C. 1997 Biomechanics: Circulation, 2nd edn. New York: Springer-Verlag.

Gervais, T., El-Ali, J., Günther, A. \& Jensen, K. F. 2006 Flow-induced deformation of shallow microfluidic channels. Lab Chip 6, 500-507.

Ghosal, S. 2002 Lubrication theory for electro-osmotic flow in a microfluidic channel of slowly varying cross-section and wall charge. J. Fluid Mech. 459, 103-128.

Gomez, M, Moulton, D. E. \& Vella, D. 2017 Passive control of viscous flow via elastic snap-through. Phys. Rev. Lett. 119, 144502.

Grotberg, J. B. \& Jensen, O. E. 2004 Biofluid mechanics in flexible tubes. Annu. Rev. Fluid Mech. 36, 121-147.

Happel, J. R. \& Brenner, H. 1983 Low Reynolds Number Hydrodynamics, 2nd edn. The Hague: Martinus Nijhoff Publishers.

Hardy, B. S., Uechi, K., Zhen, J. \& Kavehpour, H. P. 2009 The deformation of flexible PDMS microchannels under a pressure driven flow. Lab Chip 9, 935-938.

Holden, M. A., Kumar, S., Beskok, A. \& Cremer, P. S. 2003 Microfluidic diffusion diluter: bulging of PDMS microchannels under pressure-driven flow. J. Micromech. Microeng. 13, $412-418$.

Iliev, O., Mikelić, A. \& Popov, P. 2008 On upscaling certain flows in deformable porous media. Multiscale Model. Simul. 7, 93-123.

Johnson, K. L. 1985 Contact Mechanics. Cambridge: Cambridge University Press.

Johnston, I. D., McCluskey, D. K., Tan, C. K. L. \& Tracey, M. C. 2014 Mechanical characterization of bulk Sylgard 184 for microfluidics and microengineering. J. Micromech. Microeng. 24, 035017.

Katz, A. I., Chen, Y. \& Moreno, A. H. 1969 Flow through a collapsible tube: Experimental analysis and mathematical model. Biophys. J. 9, 1261-1279.

Kizilova, N., Hamadiche, M. \& Gad-El-HaK, M. 2012 Mathematical models of biofluid flows in compliant ducts. Arch. Mech. 64, 65-94.

Landau, L. D. \& Lifshitz, E. M. 1986 Theory of Elasticity, 3rd edn. Oxford: ButterworthHeinemann.

Lauga, E., Stroock, A. D. \& Stone, H. A. 2004 Three-dimensional flows in slowly varying planar geometries. Phys. Fluids 16, 3051-3062.

Lebovitz, N. R. 1982 Perturbation expansions on perturbed domains. SIAM Rev. 24, 381-400.

Lötters, J. C., Olthuis, W., Veltink, P. H. \& Bergveld, P. 1997 The mechanical properties of the rubber elastic polymer polydimethylsiloxane for sensor applications. J. Micromech. Microeng. 7, 145-147.

Love, A. E. H. 1888 The small free vibrations and deformation of a thin elastic shell. Phil. Trans. R. Soc. Lond. A 179, 491-546.

Mukherjee, U., Chakraborty, J. \& Chakraborty, S. 2013 Relaxation characteristics of a compliant microfluidic channel under electroosmotic flow. Soft Matter 9, 1562-1569.

Niu, P., Nablo, B. J., Bhadriraju, K. \& Reyes, D. R. 2017 Uncovering the contribution of microchannel deformation to impedance-based flow rate measurements. Anal. Chem. 89, $11372-11377$.

Ozsun, O., Yакнот, V. \& Ekinci, K. L. 2013 Non-invasive measurement of the pressure distribution in a deformable micro-channel. J. Fluid Mech. 734, R1.

Panda, P., Yuet, K. P., Dendukuri, D., Hatton, T. A. \& Doyle, P. S. 2009 Temporal response of an initially deflected PDMS channel. New J. Phys. 11, 115001.

Pedley, T. J. 1980 The Fluid Mechanics of Large Blood Vessels. Cambridge: Cambridge University Press.

RAJ, A. \& SEN, A. K. 2016 Flow-induced deformation of compliant microchannels and its effect on pressure-flow characteristics. Microfluid Nanofluid 20, 31.

Raj, M. K., DasGupta, S. \& Chakraborty, S. 2017 Hydrodynamics in deformable microchannels. Microfluid. Nanofluid. 21, 70.

Rubinow, S. I. \& Keller, J. B. 1972 Flow of a viscous fluid through an elastic tube with applications to blood flow. J. Theor. Biol. 34, 299-313.

SchomburG, W. K. 2011 Introduction to Microsystem Design. Berlin/Heidelberg: SpringerVerlag.

Seker, E., Leslie, D. C., Haj-Hariri, H., Landers, J. P., Utz, M. \& Begley, M. R. 2009 Nonlinear pressure-flow relationships for passive microfluidic valves. Lab Chip 9, 2691-2697. 
Small, M. K. \& Nix, W. D. 1992 Analysis of the accuracy of the bulge test in determining the mechanical properties of thin films. J. Mat. Res. 7, 1553-1563.

Sollier, E., Murray, C., Maoddi, P. \& Di Carlo, D. 2011 Rapid prototyping polymers for microfluidic devices and high pressure injections. Lab Chip 11, 3752-3765.

Squires, T. M. \& QuAke, S. R. 2005 Microfluidics: Fluid physics at the nanoliter scale. Rev. Mod. Phys. 77, 977-1026.

Stone, H. A., Stroock, A. D. \& Ajdari, A. 2004 Engineering flows in small devices: Microfluidics toward a lab-on-a-chip. Annu. Rev. Fluid Mech. 36, 381-411.

Sutera, S. P. \& Skalak, R. 1993 The history of Poiseuille's law. Annu. Rev. Fluid Mech. 25, $1-19$.

Tavakol, B., Froehlicher, G., Holmes, D. P. \& Stone, H. A. 2017 Extended lubrication theory: improved estimates of flow in channels with variable geometry. Proc. R. Soc. A 473, 20170234.

Timoshenko, S. \& Woinowsky-Krieger, S. 1959 Theory of Plates and Shells, 2nd edn. New York: McGraw-Hill.

Van Dyke, M. D. 1975 Perturbation Methods in Fluid Mechanics. Stanford, California: Parabolic Press.

Whittaker, R. J., Heil, M., Jensen, O. E. \& Waters, S. L. 2010 A rational derivation of a tube law from shell theory. Q. J. Mech. Appl. Math. 63, 465-496.

XiA, Y. \& Whitesides, G. M. 1998 Soft lithography. Annu. Rev. Mater. Sci. 28, 153-184. 